\title{
MODÉLISATION DU PANACIIE DU RHÔNE : COMPARAISON AVEC DES MESURES DE COURANTOMÉTRIE PAR RADAR VHF. ETUDE DE SENSIBILITÉ DU MODÈLE EN VUE DE L'ASSIMILATION DES DONNÉES.
}

\author{
A. BAECKEROOT, J.L. DEVENON
}

Laboratoire de Sondages Electromagnétiques de l'Environnement Terrestre, (LSEET) - UA 705 CNRS, Université de Toulon et du Var, BP 132, 83957 La Garde Cedex, France - E-mail. baeck@lseet.univ-tln.fr - jld@ lseet.univ-tln.fr

\section{Abstract}

A reduced-gravity non-linear layer model is developed in order to study river plumes. This stationnary model, based on a simpler one found in literature, considers mass and momentum exchanges in the frontal zone. Interfacial friction has been introduced to take into account the wind and underlying current effects. Supercritical flow is assumed in the outlet channel, so that characteristics method is used for numerical resolution. It provides a variable grid, strongly akin with the flow properties. For a given accuracy the number of grid points is reduced by several orders of magnitude compared with a fixed orthogonal mesh grid. This grid is felt to be optimal for implementing data assimilation. The model shows the influence of the front on the plume dynamics, and non-linear advective terms are well represented. The computed flow is comparable with measurements made with the VHF radar of the laboratory near the Rhone's mouth. The model will be improved with data assimilation, then it could be applied to other coastal effluents or coupled with a sediment transport model.

\section{INTRODUCTION}

A l'embouchure du Rhône l'eau douce, après un mélange partiel avec l'eau de mer, s'étale en surface, formant une couche d'eau saumâtre de quelques mètres d'épaisseur. La densité de l'eau du panache étant inférieure à celle de l'eau de mer du bassin récepteur, le système formé est stable. Dans le cas où il existe une vitesse relative entre les deux masses d'eau, un front se forme, caractérisé par une convergence de vitesse horizontale. La zone frontale (fig.1) est caractérisée par une forte pente de l'interface (quelques pour cent), et un mélange important avec l'eau marine. Elle s'étend sur une centaine de mètres de largeur.

Le panache du Rhône a une extension horizontale de quelques dizaines de kilomètres, une épaisseur de quelques mètres, des vitesses de quelques dizaines de centimètres par seconde donnant ainsi un écoulement surcritique vis-à-vis de la célérité locale des ondes internes. Le gradient vertical de vitesse horizontale est supposé faible devant la vitesse moyenne. La structure du panache est principalement affectée par les conditions météorologiques, les courants du bassin récepteur et la force de Coriolis pour les panaches de grande ou moyenne échelle (Buckley, 1991, Mc Climans, 1986).

Afin de prendre en compte ces par-ticularités, nous modélisons le panache par les équations non linéaires de type 'shallow-water' intégrées verticalement sur l'épaisseur du panache, avec un front simulé par une discontinuité de vitesse (Garvine, 1981). De plus des tensions interfaciales ont été introduites dans le corps du panache. La résolution est effectuée par une méthode de différences finies le long des lignes caractéristiques.

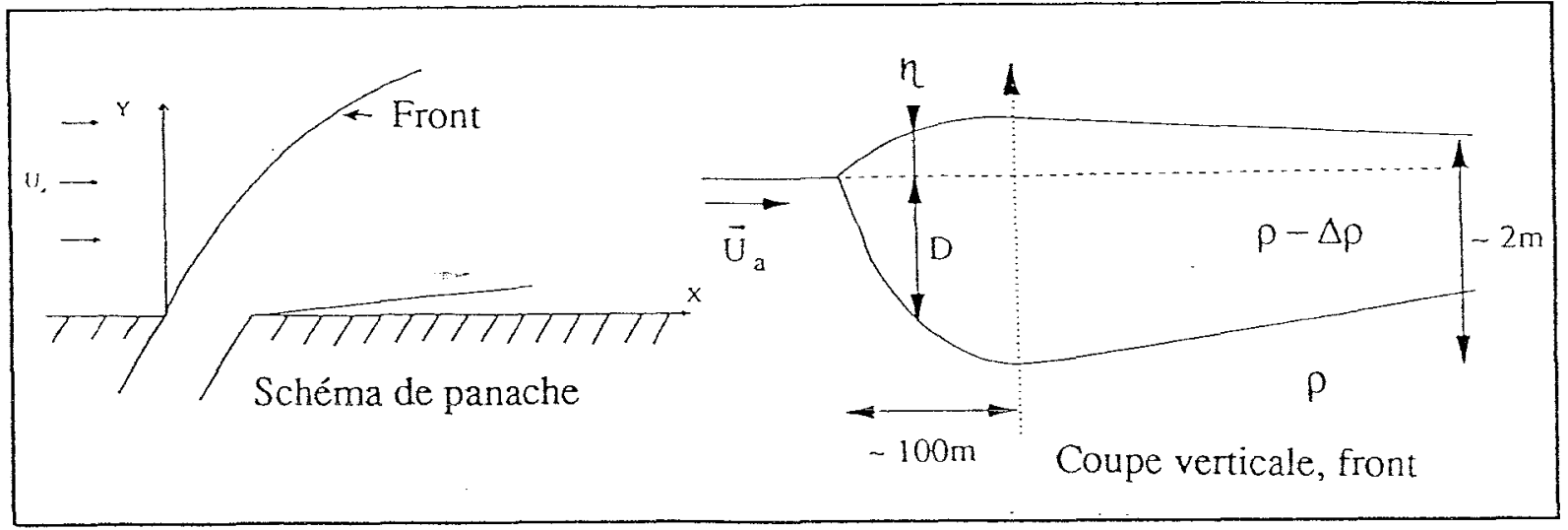

Fig. 1 : Schémas de panache 


\section{EESCRIPTION DU MODÈLE.}

\subsection{Equations.}

Le panache est modélisé en régime établi, la répartition verticale de pression est hydrostatique. La profondeur du bassin récepteur est supposée grande par rapport à l'épaisseur du panache. On fait l'hypothèse de Boussinesq, et les équations sont moyennées sur l'épaisseur du panache (Garvine, 1982) L'écoulement est alors régi par les équations suivantes :

Equation de continuité : $\vec{\nabla} \cdot(\mathrm{D} \overrightarrow{\mathrm{q}})=0$

Équation du mouvement moyen :

$$
(\vec{q} \cdot \vec{\nabla}) \vec{q}-\vec{f} \times \vec{q}=-g^{\prime} \bar{\nabla} \vec{D}+\frac{\vec{\tau}_{s}-\vec{\tau}_{b}}{\rho D}
$$

que l'on écrira aussi :

$$
\vec{\nabla}\left(\frac{q^{2}}{2}+c^{2}\right)=\frac{\vec{\tau}_{s}-\vec{\tau}_{b}}{\rho D}+(\vec{\Omega}+\vec{f}) \times \vec{q}
$$

Avec les notations suivantes:

$\eta=\Delta \rho / \rho . D$ : surélévation par rapport au niveau moyen

$\vec{q}$ : vitesse moyennée sur l'épaisseur

$\vec{\tau}:$ tension pariétale, indexée s en surface, b (bottom) au fond

$\vec{f}:$ force de Coriolis
$\vec{\Omega}=\vec{\nabla} \times \vec{q}:$ vorticité

$\mathrm{g}^{\prime}=\Delta \rho / \rho . \mathrm{g}:$ gravité réduite

$c=\sqrt{g^{\prime} D}:$ célérité des ondes internes

$\vec{k}$ : vecteur unitaire selon $\mathrm{Oz}$, verticale ascendante

En appliquant l'opérateur $\vec{k} \cdot(\vec{\nabla} \times \cdot)$ à l'équation vectorielle du mouvement moyen, on obtient à l'aide de l'équation de continuité et l'hypothèse de mouvement quasi-horizontal :

$$
\vec{q} \cdot \vec{\nabla}\left(\frac{\Omega+f}{D}\right)=\frac{1}{D} \vec{k} \cdot \overrightarrow{r o} \vec{t}\left(\frac{\vec{\tau}_{s}-\vec{\tau}_{b}}{\rho D}\right)
$$

Cette équation traduit la variation de vorticité potentielle le long d'une ligne de courant.

La stabilité verticale du système est évaluée par un nombre de Froude réduit local : $\Delta F=\frac{\left|\vec{q}-\vec{U}_{a}\right|}{c}$. Ce nombre est l'inverse du carré d'un nombre de Richardson. Pour $\Delta F>4$, le déferlement des ondes internes détruit le panache. Ce critère de stabilité sert de condition d'arrêt pour le calcul.

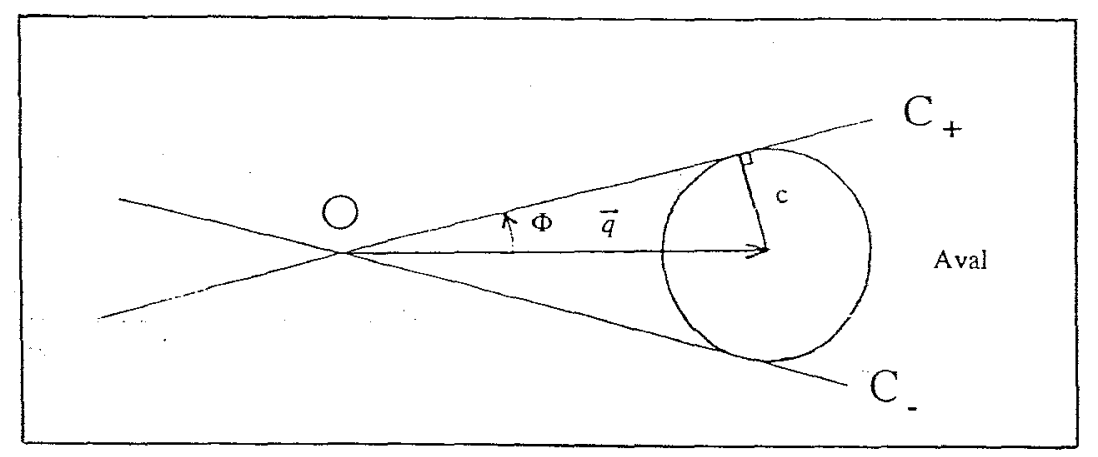

Fig. 2 : Signification des lignes caractéristiques

\subsection{Méthode des caractéristiques}

Le système d'équations à résoudre est hyperbolique, car nous considérons un écoulement surcritique (c'est-à-dire $\mathrm{q}>\mathrm{c}$ ). Nous pouvons donc utiliser la méthode des lignes caractéristiques, qui délimitent la région de l'écoulement affecté par une perturbation engendrée en un point (fig. 2).

Une perturbation initialement en $\mathrm{O}$ se propage par rapport au fluide dans toutes les directions à la célérité $\mathrm{c}$, et est simultanément entraînée avec le fluide à la vitesse $q$ de l'écoulement. Pour un écoulement surcritique l'influence des conditions en $\mathrm{O}$ est confinée dans le cône d'influence (aval), réduit à un angle limité par les deux lignes caractéristiques $\mathrm{C}+$ et $\mathrm{C}$ - (en dimension 2$)$. Le long des lignes caractéristiques, on peut écrire le système d'équations aux dérivées partielles sous forrne de différentielles totales, faisant apparaître les Invariants de Riemann (Abott, 1966). Les résultats sont analogues à ceux obtenus en aérodynamique supersonique des gaz compressibles, l'épaisseur D du panache jouant le rôle de la masse volumique du gaz.

Après calculs on obtient:

Le long de $\mathrm{C}+$ :

$$
-\sqrt{F^{2}-1} \frac{d q}{q}+d \theta=-\frac{\sqrt{F^{2}-1}}{F q} \Omega d s-\frac{\vec{q} \cdot\left(\vec{\tau}_{s}-\vec{\tau}_{b}\right)}{F \cdot c^{2} \cdot \rho \cdot D} d s
$$

Le long de C- :

$$
\sqrt{F^{2}-1} \frac{d q}{q}+d \theta=\frac{\sqrt{F^{2}-1}}{F q} \Omega d s-\frac{\bar{q} \cdot\left(\vec{\tau}_{s}-\vec{\tau}_{b}\right)}{F \cdot c^{2} \cdot \rho \cdot D} d s
$$

avec $F=q / c$ le nombre de Froude réduit, $P=\arcsin 1 / F$ I'angle de Froude, $\theta=$ angle entre Ox et $\vec{q}$

Dans le cas d'un écoulement irrotationnel et en l'absence de frottement, cas traité par R.W.Garvine, on retrouve les invariats de Riemann $J_{-}$et $J_{+}$: 
sur $C_{ \pm}: d J_{\mp}=0$, avec $\mathrm{J}_{\mp}=\theta \pm v$

où $v$ est la fonction de Prandtl-Meyer :

$$
v\left(\mathrm{~F}_{0}\right)=\int_{\mathrm{F}=1}^{\mathrm{F}_{0}} \sqrt{\mathrm{F}^{2}-1} \frac{\mathrm{dq}}{\mathrm{q}}
$$

Dans notre cas on dira donc que l'on propage des quasiinvariants le long des lignes caractéristiques.

\subsection{Méthode de résolution numérique \\ 2.3.1 Construction du maillage}

On s'appuie sur le maillage formé par les lignes caractéristiques, le long desquelles on applique les relations obtenues en 2.2 en utilisant des différences finies (fig. 3).

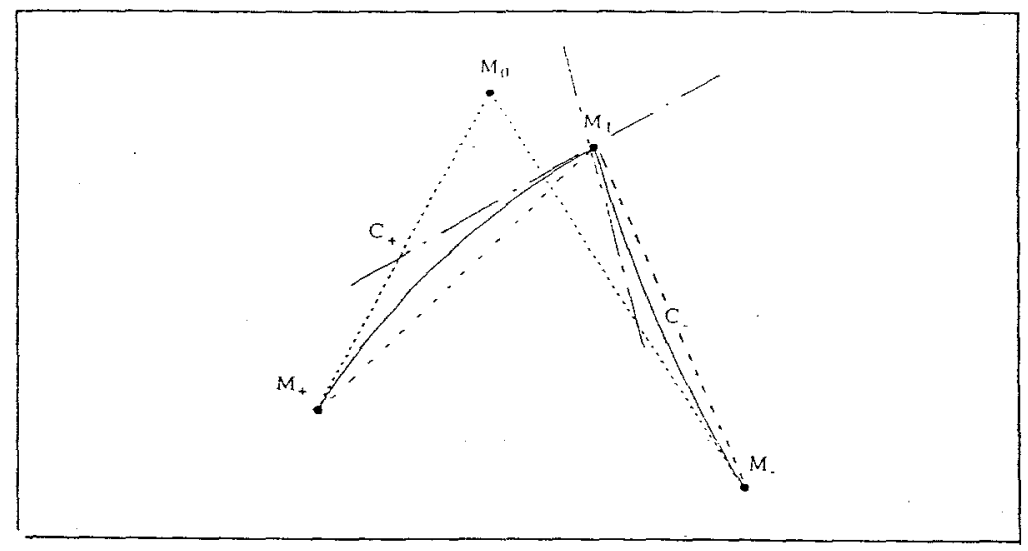

Fig. 3 : Construction du maillage

On part d'une ligne de calcul où les valeurs sont connures en chaque noeud. Les caractéristiques $\mathrm{C}_{+}$et $\mathrm{C}$. issues de deux points voisins $M_{+}$et $M_{-}$sont dans un premier temps assimilées à des segments de droites à l'échelle de la maille. Leur intersection donne une estimation de la position du nouveau noeud Mo de maillage. Les valeurs au noeud sont calculées en considérants $\mathrm{J}_{+}$et $\mathrm{J}_{-}$invariants, puis une correction due à la quasi-invariance est apportée. La position réelle $\left(\mathrm{M}_{1}\right)$ est estimée en prenant l'intersection de lignes caractéristiques moyennes ayant pour pente la moyenne de celles calculées en $M_{1}$ et de celles connues en $M_{+}$et M. . Le processus est réitéré jusqu'à stabilisation du résultat obtenu. Ainsi le maillage, qui dépend de la solution obtenue, est construit progressivement.

\subsubsection{Propriétés du maillage formé par les} lignes caractéristiques.

La répartition des noeuds de calcul est liée à l'écoulement. Les mailles sont petites dans l'embouchure où les gradients sont très élevés, et elles s'agrandissent au fur et à mesure que les gradients diminuent. Cela permet, à précision égale, de réduire considérablement le nombre de points de calcul.

La méthode des différences finies est inconditionnellement stable, car la condition de CourantFriedrich-Levy se trouve vérifiée par construction. En outre, la méthode des caractéristiques est une méthode exacte, et la propagation des quasi-invariants sur le maillage minimise les erreurs de troncature (Abott, 1966).

Toutefois il est nécessaire de réinterpoler les résultats sur une grille fixe afin de pouvoir les manipuler aisément. Ceci représente une fraction faible du temps de calcul, mais introduit une erreur licé à l'algorithme dinterpolation.

\subsection{Conditions aux limites}

Sur la côte on considère simplement l'imperméabilité de la paroi et une condition de glissement sans frottement (compatible avec l'absence de frottement à l'horizontale). La seule contrainte est donc d'annuler la composante normale de vitesse.

On montre (Garvine, 1981) qu' un front stationnaire ne peut exister qu'avec des frottements interfaciaux d'autant plus grands que le mélange est important. La région frontale est modélisée par une discontinuité de vitesse normale (condition de saut) et une paramétrisation des transferts de masse et de quantité de mouvement analogues des conditions de RankineHugoniot en aérodynamique supersonique. Les discontinuités tangentielles de vitesses ne sont pas prises en compte. Pour cela on intègre horizontalement sur toute la largeur de la zone frontale et verticalement sur l'épaisseur du panache (Garvine, 1981). Cela conduit à une description globale des échanges au front (fig.4) :

Condition de saut :

$$
\mathrm{q}_{\mathrm{f}}^{(\mathrm{n})}=-\mathrm{S} \cdot \beta \cdot \mathrm{U}_{\mathrm{a}}^{(\mathrm{n})}
$$

Condition de stationarité : $\quad C^{(n)}=\frac{U_{a}^{(n)}}{F_{a}}$;

l'exposant (n) indique la composante normale au front, $S_{e}$ caractérise l'entraînement d'une masse d'eau par l'autre. Pour $S_{e}=-1$, le transfert de masse se fait depuis le panache vers l'eau de mer. $\beta$ est un coefficient de transfert qui permet de paramétrer la discontinuité de vitesse normale à la traversée du front. Pour obtenir un front stationnaire, il faut que la célérité locale des ondes de gravité (qui est aussi la vitesse de propagation du front par rapport au fluide ambiant) compense la composante normale du courant $\mathrm{U}_{\mathrm{a}}$. On choisit donc $\mathrm{F}_{\mathrm{a}}=\mathrm{l}, \mathrm{F}_{\mathrm{a}}$ étant un nombre de Froude local au front. 


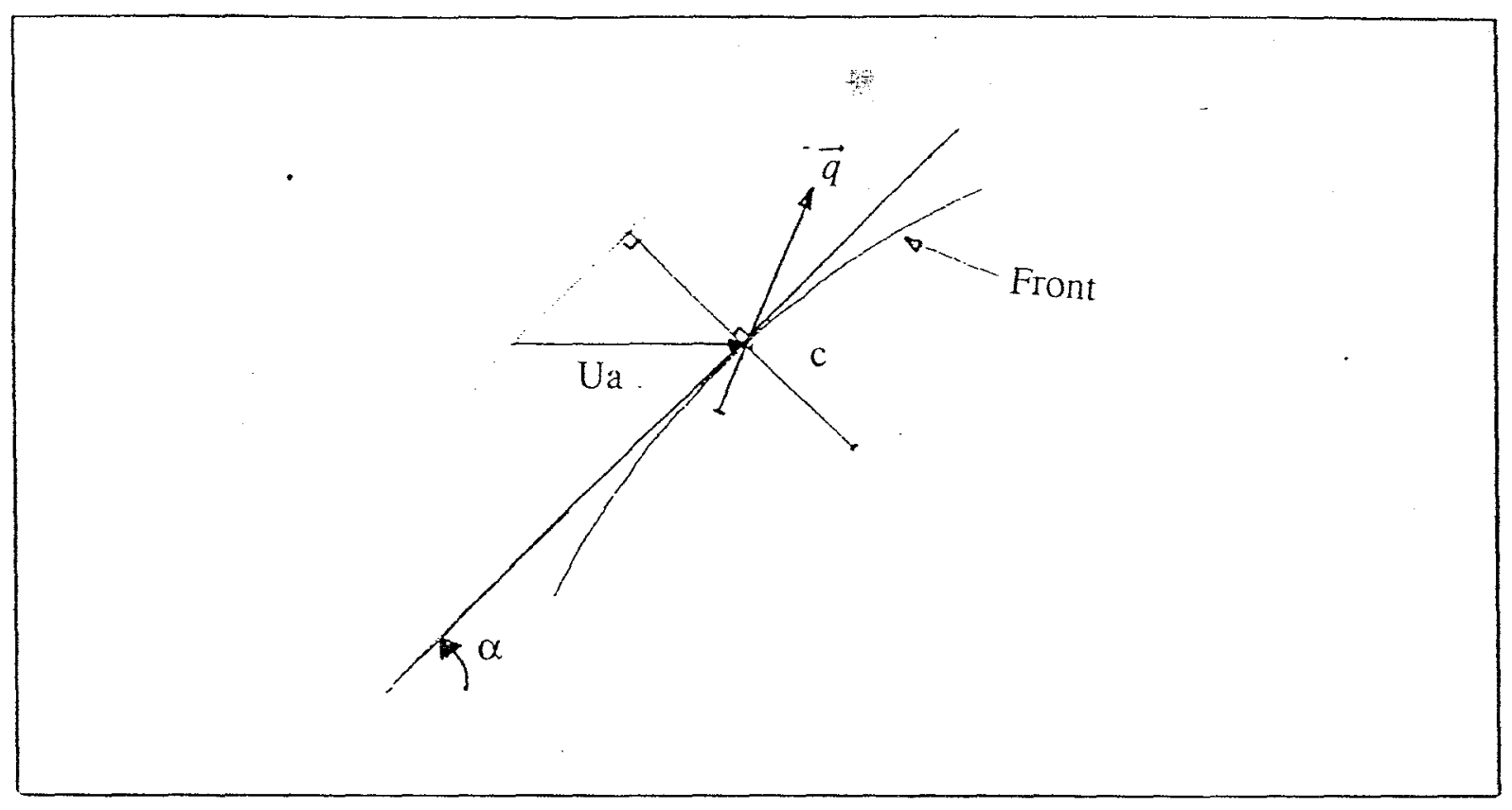

Fig. 4 : Conditions au front

Ces conditions simples permettent une bonne représentation d'un front, et les valeurs données pour les coefficients sont présentées comme celles qui foumissent les résultats les plus réalistes (Garvine, 1982). Avec ces valeurs on trouve que les caractéristiques $C_{+}$arrivent au front et șy réfléchissent pour donner des caractéristiques $C_{\text {. }}$ issues du front. Pour un point de maillage situé sur le front on dispose donc du quasi-invariant propagé par la caractéristique $\mathrm{C}_{+}$et des conditions d'échanges. Ceci établit le lien étroit existant entre la dynamique au sein du panache et la structure du front.

\section{RÉSULTATS}

La première version du modèle, sans frottement ni force de Coriolis, utilisant le maillage constitué par les lignes caractérititiques, a été validée par comparaison avec les résultats de modélisation de R.W.Garvine (1982) utilisant un maillage rectangulaire classique. La figure 5 compare les résultats obtenus. Les réseaux de lignes caractéristiques calculés par les deux modèles sont identiques, ainsi que la position du front. La mise en oeuvre des deux types de maillage a montré que, pour une même précision, le maillage caractéristique comporte de 10 à 50 fois moins de noeuds de calcul.

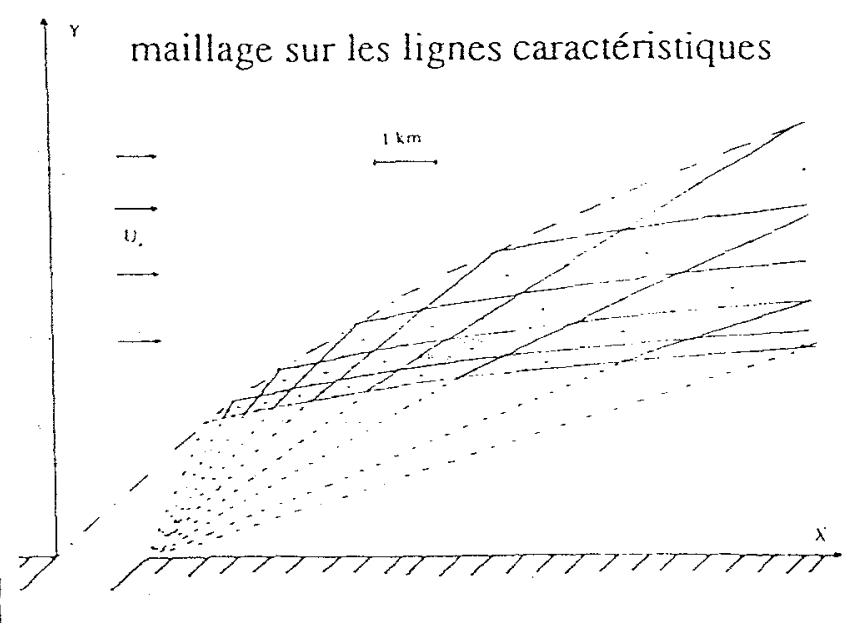

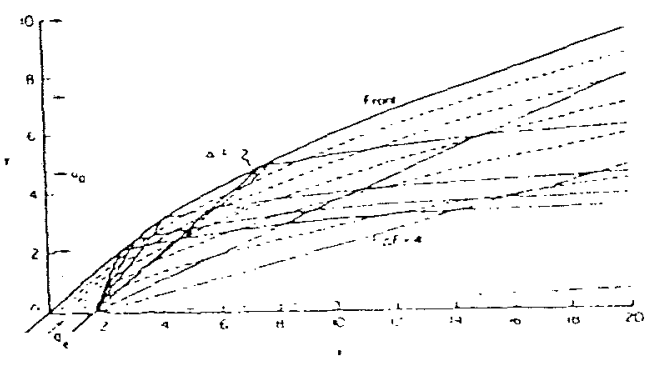

Numerical resutes for the standard case. Ambient velociry $u_{0}$ and exil velecity $\varphi_{c}$ have speds proportional to their arrou lenghts. Eight. solid lines show selected characlerisics. dashed lines shew streambines and

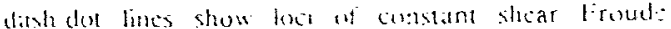
number $1 \%$ Distances shoura alome axes are normaliod by the sutce chanded whth. The exit thow angle is"

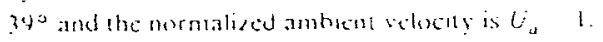

Fig. 5 : Comparaison du modèle avec celui de R.W. Garvine (1982) 
La dynamique de l'écoulement est régie par deux effets antagonistes : d'une part à l'embouchure les lignes de courants et les caractéristiques divergent ce qui correspond à une expansion du panache, d'autre part le front créé par le courant ambiant a le même effet qu'une paroi poreuse (pour la quantité de mouvement), qui comprime l'écoulement en le forçant à s'orienter dans le sens du courant extérieur.

L'introduction de frottement à l'interface eau marine / panache tend à orienter davantage l'écoulement dans le sens du courant, mais contribue aussi à dissiper de l'énergie. A l'inverse, le frottement en surface incurve les lignes de courants sous linfluence du vent et apporte de l'énergie au système. L'influence de ces deux termes est d'autant plus grande que l'épaisseur du panache est faible. Les effets des tensions interfaciales sont donc surtout sensibles à quelque distance de l'embouchure.
La force de Coriolis peut modifier de manière sensible les résultats obtenus. Proche de l'embouchure, selon les régions considérées, le mouvement est plutôt géostrophique ou plutôt inertiel, ou ne comporte pas de terme dominant. Loin de l'embouchure, comme le modèle est stationnaire, on tend à obtenir un bilan de transport de type Ekman (en considérant une tension totale surface + fond).

Globalement le vent, qui est l'élément le plus variable, impose la structure du panache modélisé. Ceci a été observé notamment lors de la campagne de mesures courantométriques par radar VHF effectuée par le laboratoire LSEET (Devenon et al., 1992). Les données collectées (une carte de courant toutes les demi-heures pendant 2 mois) couvrent une zone d'environ $30 \mathrm{~km} \times 30$ $\mathrm{km}$ au voisinage de l'embouchure du Rhône. Le front est nettement mis en évidence par la discontinuité de vitesse

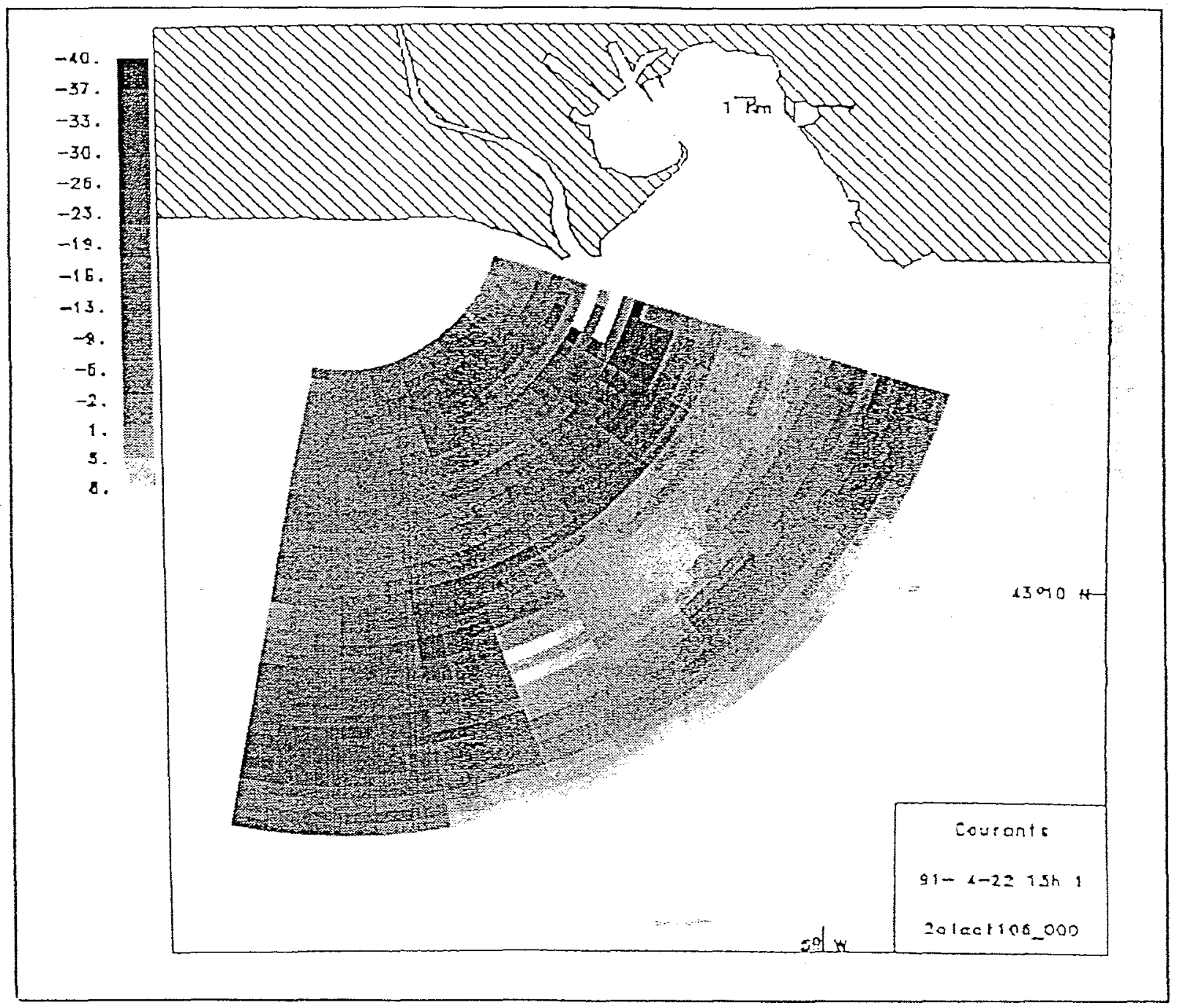

Fig. 6: Carte de vitesse (composante radiale) mesurée par radar VHF

(jusqu'a 20 à $40 \mathrm{~cm} / \mathrm{s}$ ) entre deux cellules de mesure adjacentes.

La forme générale du front est incurvée, vraisemblablement par le courant extérieur et/ou la force de Coriolis. Mais, selon les conditions météorologiques la position du front peut varier considérablement (plusieurs $\mathrm{km}$ ), ainsi que le champ de vitesse, et le panache disparait au delà d'un seuil d'agitation de la surface marine. 


\section{CONCLUSIONS ET PERSPECTIVES}

Le modèle présenté fourni des résultats morphologiquement proches des données recueillies. Sans ajustement particulier des paramètres de modélisation les dimensions horizontales et verticales du panache sont très bien estimées. Le champ de vitesses calculé est en bon accord qualitatif (en ordre de grandeur) avec l'expérience.

Le maillage caractéristique est pressenti comme étant optimal pour la réalisation ultérieure de l'assimilation des données. En effet, les notions d'observabilité et de contrôlabilité, correspondent aux cônes de dépendance et d'influence, et les lignes caractéristiques sont les mêmes que celles du modèle adjoint, ce qui devrait simplifier la mise en oeuvre de ces techniques.

En conclusion, le modèle reproduit bien les principaux traits observés sur le panache du Rhône. 11 permet de plus $\mathrm{l}^{\prime}$ analyse de cette structure complexe, et une compréhension impossible à obtenir depuis les données partielles fournies par un seul radar (seule la composante radiale de vitesse est disponible). Cependant l'assimilation des mesures effectuées permettra d'ajuster les paramètres du modèle, et permettra des déductions plus affinées. L'utilisation de la méthode des caractéristiques et du maillage naturel associé facilitent 1 interprétation physique et optimise grandement la partie numérique (le modèle ne demande que quelques minutes de calcul sur une station de travail). Il pourrait aisément être appliqué à d'autres rejets en milieu côtier, et devrait prochainement être couplé à un modèle de transport de matières en suspension (turbidité) ou de contaminants passifs.

\section{Références}

Abott M.B. 1966. An introduction to the method of caracteristics. American Elsevier Publishing Company, New York, 243pp.

Becker E. 1968. Gas dynamics, Academic Press, 297pp.

Broche P., Forget P., de Maistre J.C., Devenon J.L. and Crochet M. 1987 VHF radar for ocean surface current and sea state remote sensing, Radio Science, 22, 69-75.

Buckley A. and O'Kane J.P. 1992. A comparison of river plume models. Commission of the European Communities, Water Pollution Report, Martin J.M. and Barth H. (eds), 20, 63-73.

Devenon J.L., Broche P., de Maistre J.C., Forget P., Gaggelli J. and Rougier G. 1992. VHF measurements in the Rhône river plume, preliminary results. Water Pollution Research Reports, 2X, Proceedings of 3rd Eros Workshop, Texel, The Netherlands.

Forget P., Devenon J.L., de Maistre J.C. and Broche P. 1990. VHF remote sensing for mapping river plume circulation. Geophysical Research Letters, 17, 1097 1100 .

Garvine R.W. 1977. Observation of the motion field of the Connecticut River plume. J. Geophys. Res., 82, 441-454.

Garvine R.W. 1981. Frontal jump conditions for models of shallow buoyant surface layer hydrodynamics. Tellu.s, 33, 301-312.
Garvine R.W. 1982. A stead state model for buoyant surface plume hydrodynamics in coastal waters. Tellus 34, 293-306.

Garvine R.W. 1987. Estuary Plumes and Fronts in Shelf Waters: A Layer Model. Journal of Physical Oceanography, 17, 1877-1896.

Landau L. et Lifchitz E. 1971. Mécanique des fluides, Physique théorique, 6, Editions MIR, Moscou,

Mc Climans T.A.1986. Estuarine Fronts and River Plumes, in Physical Processes in Estuaries, J.Dronkers \& W.van Leussell (Eds.), Springer-Verlag, 560pp

O'Donell J. 1986. A numerical model of the dynamics of buoyant discharges. Ph.D. dissertation, University of Delaware, $182 \mathrm{pp}$.

O'Kane J.P. and Turner J. 1989. Preliminary results from a three-dimensional hydrodynamic model of the Rhône plume. Commission of the European Communities, Water Pollution Report, Martin J.M. and Barth H. (eds), 13, 27-43. 